\title{
PLURALIDAD DE VOCES EN LOS DISCURSOS DEL BIENESTAR: UNA EXPERIENCIA ETNOGRÁFICA REFLEXIVA
}

\author{
PLURALITY OF VOICES IN WELLBEING DISCOURSES: A REFLECTIVE \\ ETHNOGRAPHIC EXPERIENCE
}

\author{
Juan Jaime Loera González.1
}

\begin{abstract}
Este artículo reflexiona sobre el reto de visualizar la diversidad de voces y discursos de bienestar en la investigación etnográfica. Basándose en la experiencia etnográfica de trabajo de campo en dos localidades de indígenas Rarámuri en México, explora la diversidad de voces en la construcción de discursos de bienestar. El artículo, se centra en examinar dos diferentes discursos que articulan visiones de comunidad y formas de vida, y cómo éstas reflejan posiciones de dominio y marginación dentro de las localidades. Finalizo reflexionando en las formas en que los investigadores se insertan en el campo y los riesgos metodológicos de privilegiar algunas voces sobre otras en la búsqueda de nociones colectivas de bienestar.
\end{abstract}

Palabras claves: discursos; bienestar; pueblos indígenas; modos de subsistencia.

This article reflects on the challenge of visualizing the diversity of voices and discourses about wellbeing in ethnographic research. Based on an ethnographic fieldwork experience in two indigenous locations among the Rarámuri people in Mexico, the article explores the plurality of voices in building wellbeing ideas. The article focuses on examining two speeches as articulations of different visions of community and ways of living, and how they reflect positions of domination and marginalization within the indigenous people. The article concludes by reflecting on the ways in which researchers insert themselves in the field and the methodological risks of privileging some voices over others in the pursuit of identifying collective notions of wellbeing.

Key words: Discourses; wellbeing; indigenous peoples; livelihoods.

En la investigación etnográfica sobre bienestar existe un reconocimiento bien establecido sobre la diferencia entre nociones universales - occidentales y las localmente contextualizadas de bienestar (Mathews e Izquierdo 2009). Sin embargo, menos reconocida y estudiada es la diversidad de manifestaciones hacia el interior de las nociones contextualizadas de bienestar en grupos o pueblos indígenas. Aun menos estudiadas son las implicaciones y retos metodológicos presentes en el análisis y documentación de tales discursos asociados a lo que se considera la buena vida.

El presente artículo aspira ser un aporte en ese sentido. Para ello, se explora el encuentro etnográfico vivido durante una investigación cualitativa cuyo objetivo fue analizar la construcción dinámica de dichas nociones al interior de dos localidades Rarámuri: Aboreachi y Laguna de Aboreachi, ambas ubicadas en el municipio de Guachochi, en la región Tarahumara del Estado de Chihuahua al Norte de México (Loera-González 2013). El artículo pretende identificar discursos dominantes y subalternos que entretejen las múltiples maneras de entender el bienestar entre los Rarámuri de ambas localidades ${ }^{1}$. Apoyado por esta presentación de discursos de bienestar, reflexiono sobre su construcción etnográfica en trabajo de campo considerando que dichos discursos se encuentran relacionados con informantes de los cuales uno como investigador puede tener mayor interacción sobre otros. Al respecto, el artículo considera las implicaciones metodológicas en cuanto a los riesgos de pasar por alto las voces marginadas o subalternas dentro de las comunidades que visitamos como investigadores. Esto es importante porque dichas voces pueden contar una historia diferente a la expresada por los miembros más visibles -y a menudo privilegiados- al interior de las localidades. En este sentido, asumo la importancia de la reflexividad etnográfica que problematice las formas cómo los investigadores nos insertamos en el campo a fin de evitar sobre-representar una narrativa homogénea

\footnotetext{
${ }^{1}$ CONACYT, Escuela Antropología e Historia del Norte de México, Chihuahua, México. jloeragonzalez@gmail.com
} 
que podría constituir un falso sentido de comunidad al esconder la pluralidad de posiciones dentro de un grupo social (Cooke y Kothari 2001; White y Pettit 2004).

Relato cómo mi interés inquisitivo trasmutó de una búsqueda inicial de un solo discurso de bienestar coherente y bien articulado, a documentar adicionalmente varias voces disonantes y narrativas de bienestar aparentemente opuestas entre sí. La experiencia descrita implicó, por tanto, modificar técnicas metodológicas para permitir documentar al menos dos discursos de bienestar claramente diferenciados; uno enunciado de manera frecuente y abierta en ambas localidades; y un segundo que se articula de manera más oculta y privada en momentos muy concretos. Ambos discursos, más que ser concebidos como opuestos, son abordados como complementarios de lo que se presenta pública y privadamente como bienestar para los Rarámuri. Para su análisis, retomo la elaboración de James Scott (1990) sobre los hidden transcripts empleados por grupos subordinados como forma de resistencia y, los contra-discursos de Nancy Fraser (1992) como respuesta a la exclusión de espacios deliberativos que promueven mayor paridad en la participación política.

$\mathrm{El}$ artículo se divide en las siguientes secciones. Después de la introducción, describo brevemente las condiciones metodológicas en las cuales se realizó el encuentro etnográfico. El tercer apartado profundiza sobre la manera como me introduje a la localidad y las correspondientes asociaciones asumidas por mis interlocutores que influyeron en las narrativas y discursos a los que recurrentemente tenía acceso. El cuarto y quinto apartado analizan ambos discursos, identificando las voces dominantes y las voces subalternas a partir de información y extractos de entrevistas. A continuación, presento la idea de fluctuación discursiva como apoyo a una reflexión más minuciosa de las dinámicas discursivas del bienestar Rarámuri argumentando su complementariedad estratégica. Por último, presento una reflexión sobre las implicaciones metodológicas para el trabajo de campo etnográfico a partir de la evidencia presentada. Específicamente, me interesa reflexionar sobre el hecho de cómo lograr que el etnógrafo sea capaz de documentar la pluralidad de voces y, si es posible escuchar la voz del subalterno.

\section{EI Trabajo de Campo Etnográfico}

La región denominada Sierra Tarahumara consiste en una zona predominantemente montañosa que abarca por lo menos 17 municipios dentro del Estado de Chihuahua. Dicha zona es una región caracterizada por relaciones interculturales, donde además de la población no indígena, habitan cuatro grupos indígenas: Rarámuri o Tarahumara, Tepehuan, Pima y Guarijo, siendo el primero el más numeroso en términos demográficos. Según datos oficiales, el $55,14 \%$ de la población de estos municipios tiene población que se considera indígena (INEGI 2015). Dicha población indígena muestra un patrón de asentamiento espacialmente disperso donde el 78,2\% vive en localidades menores a 2.500 habitantes $^{2}$ (INEGI 2015). Además de ser un espacio intercultural, la Sierra Tarahumara es considerada como una de las zonas indígenas con mayor desigualdad entre población indígena y no indígena en México según el Índice de Desarrollo Humano para los Pueblos Indígenas (PNUD 2010). Dicho Índice ubica a los seis municipios con menor Desarrollo Humano de México dentro de la Sierra Tarahumara. Reflejo de dicha desigualdad es la proliferación en las últimas décadas en el número e intensidad de conflictos socioambientales debido al incremento de la extracción y explotación de recursos energéticos, forestales, y mineros, que distribuyen los beneficios de manera desigual (Toledo 2015).

La investigación etnográfica se realizó en dos localidades del Estado de Chihuahua en el Municipio de Guachochi: Aboreachi con una población completamente Rarámuri (120 habitantes); y Laguna de Aboreachi con una población mayoritariamente mestiza ( 315 habitantes) ${ }^{3}$. El periodo de campo se llevó a cabo de enero a diciembre de 2010 teniendo como objetivo desarrollar una etnografía que documente distintas voces sobre bienestar, aspiraciones y entendimientos que forman una buena vida para el pueblo Rarámuri. Para complementar la investigación etnográfica apliqué encuestas a 30 individuos autoidentificados como Rarámuri y 30 habitantes mestizos de las localidades seleccionadas. El trabajo de campo implicó vivir en ambas comunidades por periodos prolongados permitiéndome conocer los modos de subsistencia de la agricultura de autoconsumo. Para ello, la observación participante fue muy útil en el contexto de actividades festivas y faenas colectivas. Entre los Rarámuri, es frecuente la presencia de teswuino -bebida muy apreciada elaborada a partir de maíz fermentado- en las celebraciones religiosas o faenas de trabajo. Estas reuniones arraigadas en las tradiciones y constituyen una parte importante de las redes de cooperación social que conforman familiares y amigos cercanos (Kennedy 1963, 1978; Saucedo 2003; Urteaga 1998)4. Durante estas reuniones, pasé tiempo escuchando y participando en conversaciones colectivas y en diálogos privados, conociendo trayectorias personales, corroborando información e identifiqué versiones contradictorias.

El trabajo de campo fue inspirado en gran medida por Stephen Gudeman y Alberto Rivera (1990) en su trabajo titulado Conversaciones en Colombia. 
De igual manera que ellos, ambicioné construir una comunidad de conversación con los informantes para indagar la vida cotidiana, no solo para obtener información desde una perspectiva local, sino también como una forma de verificar y validar las fuentes de información. Esto se hizo para tratar de evitar las habituales asimetrías que se gestan en la investigación de campo (Fals-Borda y Rahman 1991). En este sentido, el investigador se posiciona como parte del mismo espacio estudiado, vinculándose a subjetividades y asociaciones preexistentes de los interlocutores en las localidades. Durante mi estadía en las localidades, la reflexividad fue clave para los objetivos de investigación. Es decir, constantemente reflexionaba sobre mi papel como etnógrafo, las suposiciones y expectativas de la población local hacia mi presencia, y sobre las ideas locales de bienestar. A menudo, reflexionaba sobre estos temas de manera individual, por ejemplo, mientras escribía mi diario etnográfico en las noches, y en otras ocasiones de forma colectiva con mis interlocutores validando información registrada anteriormente ${ }^{5}$.

De igual manera, el proceso de recopilación de información implicó reconocerme como parte de un contexto previo y, por tanto, necesité adaptarme a él. Por ejemplo, aprendí que entre los Rarámuri hacer preguntas de manera directa y persistente no se considera un gesto amable. Al hacerlo, empecé a usar mis propios conceptos y términos en entrevistas y conversaciones, tales como bienestar, felicidad o buena vida, y posteriormente -como se describe más adelante- la gente comenzó elaborar y compartir conmigo sus propias ideas de lo que es una buena vida en sus propios términos.

Sin embargo, durante el transcurso del trabajo de campo poco a poco se hizo evidente un hecho que no había considerado previamente en su etapa inicial. Al avanzar el encuentro etnográfico era indudable que mantenía contacto con un perfil limitado de individuos que fungían como interlocutores seleccionados por y desde liderazgos indígenas. Esto se hizo visible, por ejemplo, cuando en las localidades se promovía constantemente entrevistarme y conversar con individuos considerados como conocedores y garantes de la cultura Rarámuri. Entre ellos, se encontraban personas respetadas que gozaban de cierto estatus y prestigio, la gran mayoría habían ocupado cargos en el sistema político Rarámuri ya sea en ese momento o en el pasado reciente. Es decir, eran voces consideradas como legítimas y autorizadas para ser elocuentes interlocutores de "lo Rarámuri" y por ende de las nociones de bienestar propias para dicho pueblo indígena. En otras palabras, estaba ante un discurso dominante que tendía a subrayar representaciones étnicas homogeneizadas y que enfatiza las diferencias entre lo Rarámuri y lo no-Rarámuri.
Como profundizo en el apartado siguiente, el adoptar una posición reflexiva constante durante el trabajo de campo me permitió darme cuenta de la pluralidad discursiva de ideas de bienestar en el contexto Rarámuri.

\section{Aproximación a la Diversidad de Discursos}

Al tener experiencia previa en trabajo de campo en la región del norte de México me introduje a las comunidades Rarámuri de la forma como muchos antropólogos -incluyéndome- se habían aproximado a la región con anterioridad; a través, primeramente de contactarse con el Seriame -líder político tradicional entre los Rarámuri-. En otras palabras, me introduje en las localidades a través de los canales tradicionales de autoridad y fue a través de esos canales que obtuve permiso para realizar el trabajo de campo.

En consonancia con la práctica habitual, mi primer contacto se produjo en la forma de una visita informal a la casa del Seriame de Aboreachi. Después de presentarme, mis intenciones y presencia en la comunidad fueron explicadas junto con los objetivos fundamentales de la investigación. El siguiente paso fue mi presentación, encabezada por el Seriame, al resto de los habitantes de la localidad reunidos en ocasión festiva durante un día domingo en el patio de la iglesia de Aboreachi. Esta primera presentación fue una oportunidad útil que me permitió explicar públicamente mi presencia y objetivos sobre mi trabajo. A raíz de esta presentación, mi presencia y motivos fue conocida por los habitantes de Aboreachi. Sin embargo, la asociación pública de mi persona con la figura de autoridad tradicional era evidente al estar literalmente al costado próximo del Seriame durante el evento público. La presentación por la autoridad era al mismo tiempo un acto de legitimación de mi parte y un posicionamiento que públicamente me asociaba con el Seriame y al poder político que representa.

Esta primera asociación con la figura de autoridad fue muy importante pues repercutió en el tipo de información que tendría más accesible durante el transcurso del trabajo etnográfico, por lo menos durante una primera fase. Por un lado, esta primera aproximación a los habitantes de la localidad representó la oportunidad de conocer otros individuos y establecer las condiciones para crear confianza entre los hombres y mujeres cuyas percepciones estaba tratando de documentar. Por otro lado, sin embargo, esta asociación me concedió inadvertidamente un mayor acceso a los informantes en posiciones de poder dentro de la localidad Rarámuri, dejando al margen aquellos miembros de la comunidad que no eran cercanos al liderazgo político Rarámuri. A continuación, analizo las diferentes voces encontradas en el campo. 


\section{Voces Dominantes}

Mis primeros informantes durante las etapas iniciales del trabajo de campo fueron hombres y mujeres que disfrutaban de un alto estatus social, la mayoría era parte del sistema político Rarámuri ${ }^{6}$. Ellos a su vez me presentaron a gente con un perfil muy similar y que expresaron una idea de bienestar común a la expresada por ellos mismos. Es decir, sus narraciones sobre qué era y cómo se obtenía el bienestar para los Rarámuri eran homogéneamente construidas. Dicho discurso de bienestar habla de la revitalización de medios de subsistencia Rarámuri a través del fortalecimiento de prácticas culturales (fiestas ceremoniales del ciclo agrícola, redes de solidaridad basadas en el teswuino, formas de convivencia, estructuras de autoridad). Dichos medios de subsistencia se presentan como parte del discurso dominante de tal manera que define las formas de vida Rarámuri y las difiere de las no-Rarámuri. El discurso exhibido por dichos individuos, se enmarca en términos normativos en la idea de "vivir en el camino correcto'. Al paso del tiempo, entendí que 'vivir en el camino correcto' atañe a los imaginarios social y culturalmente deseables de la normatividad Rarámuri que hace visibles las diferencias entre los Rarámuri y los no Rarámuri en términos de medios de subsistencia (Loera González 2014).

En cierto sentido, este discurso se basa en el deseo y el ideal de homogeneidad en las condiciones de vida e implica necesariamente la capacidad colectiva para controlar el destino de sus propias prácticas culturales y espacios políticos. Para los portadores de este discurso, la búsqueda de bienestar implica mantener condiciones de vida que permitan vivir bien; esto no implica necesariamente mejorar dichas condiciones desde una perspectiva monetaria o consumista vinculado a los supuestos de la sociedad de mercado. Si bien no se oculta a nivel enunciativo y práctico las ventajas de la mercantilización creciente en la vida cotidiana, ciertamente sí se descarta tajantemente la necesidad imperiosa de la acumulación material y económica.

Hay dos dimensiones visibles en este primer discurso de bienestar; (a) la capacidad de subsistir con la agricultura de subsistencia, y (b) la importancia de las redes de solidaridad en las comunidades de origen. Ambas sugieren que para un importante segmento de los Rarámuri una idea de bienestar se alcanza mediante medios tradicionales de subsistencia como la agricultura familiar a pequeña escala; medios de producción comunitarios en lugar de la propiedad individual; sistemas sociales que dependen en gran medida de la convivencia y cooperación entre familias y vecinos; y las formas de distribución y el intercambio recíproco que suponen beneficios colectivos más que individuales.
Los siguientes extractos de entrevistas ejemplifican ambas dimensiones:

Si un hogar Rarámuri trabaja su parcela de tierra bien, es decir, de acuerdo con las tradiciones de nosotros y como los de antes [antepasados] solían hacer, entonces va a estar bien y no tendrá pobreza (entrevista Laguna de Aboreachi, México, julio de 2010).

Mientras tengamos tierra para trabajar y dejar como herencia a mis hijos no hay problema de pobreza, con el maíz es suficiente, es la base para tener pinole, teswuino y tortillas; el alimento se puede complementar con verduras que juntemos estacionalmente como los quelites y hongos [...] Y si usted tiene amigos y familiares que le ayuden a sembrar es algo muy bueno. Por eso es que tenemos que hacer teswuino ofrecerlo a Tatadiosi [Dios] y a sus amigos para ayudarlo cuando lo necesite [...] Si usted tiene dinero y una gran cantidad de posesiones otras personas tendrán envidia y eso no es bueno, ellos le dirán que usted no necesita ayuda porque tiene demasiadas cosas (entrevista Aboreachi, México, junio de 2010).

Este primer discurso también hace hincapié en exteriorizar un imaginario de homogeneidad entre las familias de las localidades. Los sujetos entrevistados asociados a las voces dominantes tienden a afirmar que todos los hogares de la localidad son influidos por las mismas condiciones y factores. Destacan, como ilustración, las duras condiciones climatológicas que afectan todos los cultivos por igual. Sin embargo, ciertas diferencias entre los sujetos asociados a las voces dominantes y las voces subalternas se escapan de ser enunciados públicamente. Por ejemplo, además de las diferencias en cuanto a la participación en los puestos políticos Rarámuri, una diferencia evidente entre miembros asociados a voces subalternas es que suelen ser jóvenes adolescentes o en matrimonios recién formados muchos de ellos privados de acceso a tierras y parcelas propias para practicar la agricultura de autoconsumo. Esto no suele ocurrir entre los Rarámuri que han ejercido algún cargo en el gobierno tradicional. Aun cuando las diferencias resultaban ser evidentes de hogar a hogar en el tamaño de la superficie cultivable o incluso, en la capacidad de enfrentar shocks, una porción importante de autoridades indígenas opta por no reconocer o expresar abiertamente estas diferencias. Es decir, se da mayor importancia a las condiciones comunes y colectivas que deben primar en lugar de circunstancias que impliquen diferenciación: 
Aquí [en Aboreachi], todos vivimos lo mismo y sufrimos de las mismas cosas (...) no es como en Chihuahua [ciudad] o en otros lugares. Si alguien necesita algo, hay que ayudarle, ya que podrían ser nosotros mismos los que estemos en esa situación mañana (entrevista Aboreachi, México, febrero de 2010).

Esta tendencia a expresar uniformidad colectiva refleja aspectos de un ideal social que se hace visible en este discurso asociado a cierto imaginario de identidad étnica Rarámuri, es decir, a la idea de ser Rarámuri como integrantes de una misma comunidad homogénea social y económica. Ejemplo de ello es el siguiente extracto:

Todos nosotros estamos emparentados de alguna manera y nos apoyamos; compartimos herramientas agrícolas o a veces animales para nuestras parcelas. Eso nos hace a todos Rarámuri [...] Si no llueve sufrimos todos por igual; si llueve los cultivos crecen bien para todos. Así se vive aquí (entrevista Aboreachi, México, febrero de 2010).

El énfasis en la experiencia compartida también nutre el sentido de cohesión comunitaria y pretende evitar la confrontación, el resentimiento o la envidia. Para las autoridades tradicionales dirimir conflictos internos es de gran importancia para conservar su estatus dentro de la comunidad. Al igual que otros pueblos indígenas, los Rarámuri reconocen que la envidia y resentimiento que originan individuos por jactarse de ser relativamente más acomodados que el resto o hacer alarde de los bienes materiales produce un efecto negativo que pone en peligro el sentido de cohesión y homogeneidad dentro de la localidad.

Dios quiere que vivamos felizmente sin conflicto; si seguimos peleando, gritando y portándonos mal en las teswuinadas - como hacen algunos jóvenes adolescentes - creamos problemas para todos (entrevista Laguna de Aboreachi, México, junio de 2010).

Evidentemente, la percepción de homogeneidad social no es compartida por todos en Aboreachi. Algunos Rarámuri asumen una voz más oculta y menos presente en espacios públicos donde enfatizan las condiciones de vidas desiguales entre sus vecinos, sugiriendo que la cohesión es una representación idealizada de la vida en Aboreachi y Laguna de Aboreachi. Como sostiene Li (1996:502), este tipo de representaciones idealizadas de comunidad destacando la armonía, igualdad y tradición permiten la construcción de una serie de estrategias propicias para obtener o mantener acceso a recursos que la comunidad designa como imprescindibles. Más allá de esta afirmación estrictamente instrumental, el imaginario de comunidad ideal proporciona un vocabulario de legitimación de sistemas de poder vigentes.

$\mathrm{Al}$ encontrarme con este primer discurso de bienestar durante el trabajo de campo en la Sierra Tarahumara, era atractivo documentarlo como la única construcción colectivamente compartida de bienestar entre los Rarámuri. Era atractivo porque el discurso parecía unificar y presentar su identidad étnica como un instrumento político dispuesto y ordenado. Además, tenía clara resonancia con una construcción colectiva del bienestar e identidad étnica enmarcada por las largas luchas políticas de los pueblos indígenas en América Latina. Sin embargo, en mi papel de etnógrafo tenía la responsabilidad de profundizar y examinar las diversas construcciones del bienestar escuchando aquellas voces que surgen de manera más tímida y oculta, alejadas de los espacios de poder local y que pueden proporcionar nuevos matices a la comprensión de las nociones del bienestar.

En el siguiente apartado exhibo evidencias de otras voces que se posicionan de manera diferente al discurso hasta ahora planteado que expresaban ideales de vida y entendimientos del bienestar de manera menos pública. Me refiero a esas voces como un discurso subalterno ya que ocupan una posición subordinada dentro del contexto Rarámuri de la misma manera que Fraser (1992:123) utiliza el término 'contra-discurso' para designar interpretaciones de oposición al discurso dominante.

\section{Voces Subalternas}

De manera general, estas voces expresan la aspiración y el deseo de vivir una vida similar a la mostrada por la sociedad no-Rarámuri. Dichas voces eran activadas por individuos que destacan sobretodo la importancia de asegurar un ingreso familiar en lugar de depender de la agricultura de subsistencia. Como se mencionó anteriormente, dichas voces son enunciadas de manera más clara y cotidiana por jóvenes y matrimonios recientes que no suelen tener acceso a parcelas cultivables o tierras suficientes para practicar la agricultura de autoconsumo, uno de los elementos fundamentales que compone el primer discurso. Una particularidad es que en su mayoría eran jóvenes con experiencia de trabajo fuera de la comunidad laborando temporalmente en el sector de manufactura, o como trabajadores eventuales en campos agrícolas en ciudades o estados próximos. Esta práctica de migración temporal es común en la zona de la Tarahumara, de hecho es una práctica esperada 
entre los jóvenes Rarámuri varones y mujeres pues constituye una opción económica accesible.

Justificados por las condiciones de precariedad e inseguridad alimentaria que implica la dependencia del cultivo agrícola como forma primaria de subsistencia, estas voces suelen ser más abiertas a considerar la posibilidad de emigrar a una ciudad cercana en busca de un empleo como factor para tener bienestar. De igual manera, estas voces frecuentemente incluían el deseo de poseer bienes materiales y la expectativa de mejorar sus condiciones de vida, ya sea teniendo un vehículo vistoso, más cantidad de ropa, o procurar la construcción de una extensión de su hogar. En parte, se puede argumentar que las relaciones de desigualdad interétnicas en ámbitos económicos, sociales y políticos existentes entre la población no indígena y los Rarámuri en la Sierra Tarahumara tiene el efecto de provocar demandas de distribución de oportunidades laborales, y acceso a recursos y servicios por encima de demandas de reconocimiento de distinción cultural para los Rarámuri.

Dichas voces aquí exploradas así como otras experiencias similares documentadas en el trabajo de campo, guardan ciertas semejanzas a lo que Scott (1990) llama el "discurso público" y el "discurso oculto" en el contexto de las relaciones entre poblaciones campesinas y minorías étnicas en Asia. Por "discurso público" Scott entiende las relaciones explícitas que se dan entre subordinados y detentadores de poder. En dicha relación además de prácticas de dominación evidentes, se crea un espacio social donde deseos, motivaciones y subjetividades salen en escena transmitiendo lo que el grupo dirigente asume de ellos. Sin embargo, dicho espacio no ocurre sin conflicto ni resistencias pues los sujetos subordinados desarrollan técnicas como el manejo de la apariencia, silencios, códigos, y gestos que ellos controlan. Este "discurso oculto" es considerado por Scott como la conducta fuera de escena que confirma, contradice o tergiversa lo que aparece en el discurso público. Es decir, el "discurso público" es el discurso formado por el grupo dominante activado abiertamente a cualquier observador, y el "discurso oculto" el que se gesta como el hidden transcript fuera del alcance del grupo dominante.

No fui alentado por las figuras de poder de Aboreachi y Laguna de Aboreachi para hablar con estas personas acerca de cómo un Rarámuri debe vivir o sus percepciones en torno al bienestar. Frecuentemente, los jóvenes que migraban, las personas que no participaban tan visible y activamente en las fiestas colectivas, o las que protagonizaban conflictos en las teswuinadas eran referidos como "no suficientemente Rarámuri'. Estos individuos no eran considerados como interlocutores para hablar sobre el bienestar
Rarámuri en los ojos de las personas que movilizaban el primer discurso, y por tanto sus experiencias son desechadas por un sector de la población local.

En consecuencia, empecé a visitar los hogares de la comunidad designados como los 'no suficientemente Rarámuri' y a entablar conversaciones uno-a-uno con estos sujetos que al inicio de la investigación no fueron considerados informantes clave. El hecho de visitarlos en sus hogares y tener un contacto más individual permitió que, con el tiempo, surgiera confianza para hablar con más libertad que en áreas públicas y visibles de las localidades. En el caso de los adolescentes y jóvenes que emigran estacionalmente para trabajar en los campos agrícolas o maquiladoras, regresaban a sus hogares con nuevas experiencias y adquisiciones materiales que al exteriorizarse en la comunidad los hacía vulnerables a acusaciones de crear envidia. En otros casos, algunos de estos individuos habían sido acusados de reñir en teswuinadas o festividades colectivas y por lo tanto se les acusaba de alterar las normas sociales de la comunidad ${ }^{7}$.

En este sentido, dichas voces expresan más abiertamente la necesidad de reclamar igualdad de oportunidades para acceder a mercados laborales disponibles en ciudades, y en cierto modo, reclaman una política redistributiva más que de reconocimiento étnico(Fraser 1997). Mientras que el discursodominante Rarámuri evoca la suficiencia de la agricultura familiar como modo de subsistencia, el discurso subalterno destaca su insuficiencia y sus limitaciones para obtener bienestar. Encontrar una fuente de ingresos alternativa por tanto se convierte en una prioridad. La siguiente experiencia proporciona un ejemplo.

Aquí en Aboreachi, uno tiene tan pocos deseos y antojos; por ejemplo, uno puede ser feliz con simplemente disfrutar de una Coca-Cola que compró en la tienda - o si alguien te la regala, pues mejor! [...] Pero a veces me aburro, no hay nada aquí, así que tengo que trabajar en la ciudad, para ganar dinero para la familia, este año no hicimos la mitad de la cosecha de lo que necesitamos [...] Yo sería feliz si tan sólo pudiera pagar todas mis deudas y tener dinero para poder comprar más cosas, por eso Oportunidades [programa de protección social] es importante, tal vez con el tiempo me puedo mover a la ciudad y hacer mi vida allí (entrevista Aboreachi, México, octubre de 2010).

Este testimonio de un joven Rarámuri muestra cómo, para algunos, sus aspiraciones los apartan de las voces dominantes expuestas anteriormente. Considera a la comunidad no como parte de un sistema de tradiciones y formas de vida viables, sino un lugar de 
aburrimiento y ausencias. De forma preponderante se manifiesta la aspiración/necesidad de obtener un ingreso. De pronto, la migración se convierte en una opción. Una opción que por un lado permite una cierta autonomía económica y por otro permite la posibilidad de vivir fuera de las directrices de sus mayores.

Trabajando en la cosecha del melón es una buena opción, vienen desde lejos para buscar a gente que quiera trabajar [...] ya van dos veces en que yo y mis primos vamos para allá. A ganar dinero y gastarlo también. Espero que con el tiempo pueda ahorrar para irme a vivir a otro lado, aquí no hay nada (entrevista Laguna de Aboreachi, México, julio de 2010).

Yo me quiero ir de aquí [Aboreachi] porque quiero seguir estudiando, aquí solamente hay escuela hasta la secundaria y prepa en la Laguna pero eso es todo. Si uno quiere estudiar más se tiene que ir, pues yo quiero estudiar y no quedarme aquí como mis papas, yo quiero vivir mejor (entrevista Aboreachi, México, septiembre de 2010).

En lugar de un énfasis en la tradición común y la buena vida, los jóvenes hacen hincapié en la esperanza de un futuro mejor para ellos y sus familias. La experiencia de trabajar, al menos temporalmente, trae aspiraciones de nuevas posibilidades más allá del contexto de escasez en la región Tarahumara y -se podría argumentar- fuera de los valores normativos que desalientan la acumulación material y promueven el comunitarismo, elementos básicos del discurso visto antes. Con ello, otra vida es imaginada como un medio para obtener beneficios potenciales, tanto a nivel individual como colectivo. Así, la migración parece constituirse como una transición crucial en el cálculo del bienestar con el que principalmente -aunque no exclusivamente- los jóvenes se identifican (Chindarkar 2014; Wright 2012).

De esta manera, el bienestar implica una encrucijada de opciones, decisiones y aspiraciones que en buena medida se nutren del contexto y entorno donde florecen. Las formas de sustento practicadas por un sector Rarámuri de Aboreachi y de otras localidades, basadas en una combinación de estrategias económicas no encajan fácilmente con las formas de sustento de las grandes metrópolis. La encrucijada de opciones y decisiones se refiere a que los sujetos deben de ocultar sus aspiraciones de vida del escrutinio de autoridades locales ya que sus aspiraciones no corresponden fácilmente a las representaciones e imaginarios del sector que ostenta el poder político. Por lo tanto, dicho discurso se ubica en una posición subalterna a las ideas de bienestar expresadas de manera más abierta por las autoridades Rarámuri.

\section{Dando Sentido a Ambas Voces}

Resalto que la discordancia de voces encontradas es parte de la complejidad de la vida Rarámuri. El enfrentamiento discursivo mostrado refleja una lucha por una representación de la identidad cultural de los Rarámuri sobre la vida misma y cómo ésta debería ser: ¿Cuáles son las luchas de representación e imaginarios convocados en la significación del bienestar? Por una parte, las voces dominantes se articulan en un discurso que habla de una identidad que reclama el reconocimiento y una diferencia estratégicamente homogeneizada e ideal (Spivak 1987). En cierta manera, dibuja paralelismos al discurso político del Buen Vivir posicionado por pueblos andinos de América Latina en el sentido que, en algunas de sus expresiones, retoma la necesidad de rescatar conocimientos y prácticas relativos a formas de vida propias de los pueblos indígenas (Gudynas 2011; Huanacuni Mamani 2010). Dichas formas de vida hablan del derecho al reconocimiento de prioridades y aspiraciones apartadas de la sociedad global e ideas hegemónicas de bienestar ${ }^{8}$. Por otra parte, las voces disidentes representan un discurso que hace hincapié en la necesidad de asegurar el sustento basados en una apertura de oportunidades socioeconómicas disponibles, sean estas alineadas o no a las aspiraciones de los liderazgos políticos Rarámuri. Por lo tanto, estas voces se vinculan a las demandas colectivas por la redistribución de oportunidades económicas dentro del Estado mexicano y por una participación democrática más amplia.

Los testimonios aquí presentados pueden ser leídos en términos de las categorías de "discurso público" y "discurso oculto" de Scott (1990) ya que son útiles para ilustrar la relación de poder de ambos conjuntos de voces y los diferentes escenarios donde se hacen presentes. De igual manera, son aplicables las definiciones de Fraser (1992) sobre espacios contra-públicos pues dichos espacios representan entidades discursivas paralelas donde los miembros de grupos sociales inventan y circulan discursos y contra discursos, que a su vez les permiten formular interpretaciones de sus identidades, intereses y, para el caso de los Rarámuri, el bienestar.

Sin embargo, las luchas entre voces dominantes y subalternas se activan en formas complejas y no implican posiciones absolutas. No es que las voces subalternas articulan una visión absolutamente contraria a los medios de subsistencia basados en una agricultura a pequeña escala, redes de solidaridad y cooperación como elementos constitutivos del discurso dominante y público, sino que apela más bien a la vulnerabilidad económica implícita en ellas. Fraser (1992) considera que los contra-discursos 
pueden compensar parcialmente, aunque no erradicar en su totalidad, los privilegios políticos y económicos que disfrutan los miembros de los grupos sociales dominantes en las sociedades estratificadas. Para el caso de los Rarámuri, los portadores del contradiscurso armados con concepciones diferentes de vida y bienestar, pueden enfrentar su nivel de desventaja de participación política al interior del grupo apelando a otros intereses y necesidades en sus interlocuciones con sujetos políticamente dominantes Por ejemplo, justificando su necesidad de ausentarse de las localidades para trabajar como migrantes temporales.

Si consideramos la complejidad de las relaciones inter-étnicas con la población no indígena que rodea al pueblo Rarámuri, ambos discursos no están necesariamente en oposición uno con el otro, sino más bien su relación puede ser descrita más precisamente como estratégicamente complementaria en su relación con el sector dominante y mestizo. En algunos momentos. Moviéndose estratégicamente entre el espacio público y lo oculto (Scott 1990) y en función de espacios específicos, los Rarámuri enuncian con mayor elocuencia e intensidad algún elemento sobre otros sin descartar por completo ambos discursos. Con ello, el pueblo Rarámuri tiene a su disposición recursos de dos aparatos discursivos para hacer frente a las relaciones de poder. Por una parte, hacen hincapié en demandar una distribución equitativa de derechos sociales con respecto a la sociedad dominante, por ejemplo, pueden enfatizar la demanda de tener acceso a servicios públicos digno a la par de localidades no indígenas. Por otra parte, pueden hacen uso de un discurso que hace hincapié en la necesidad de reconocer particularidades culturales en su forma de vida como indígenas, por ejemplo, en la defensa de una modo de vida basado en la agricultura de autoconsumo. Estos dos aparatos discursivos se hacen existir en momentos y espacios interétnicos ya sea en encuentros con funcionarios públicos gubernamentales en sus localidades de origen o en oficinas en los centros urbanos de poder político.

En este sentido, argumento que la idea de bienestar entre los Rarámuri puede ser entendida como una fluctuación dinámica que busca el equilibrio entre dos fuerzas: el discurso de los derechos colectivos de reconocimiento, y el discurso de los derechos de distribución (Fraser 1997). De esta manera argumento que en su relaciones al interior del grupo, muestran dos discursos claramente definidos, uno oculto-subalterno y otro público-dominante, pero que hacia el exterior, en sus relaciones con la sociedad mexicana, ambos discursos pueden ser transitados y articulados para nivelar relaciones de poder asimétricas. Por tanto, los resultados aquí presentados atienden a las diferencias hacia el interior del grupo; dos discursos concretos y diferenciados. Sin embargo, hacia la sociedad nacional, individuos Rarámuri usan ambas caminos de bienestar de forma complementaria.

Por un lado, un bienestar asociado al derecho colectivo a mantener una subsistencia basada en la agricultura familiar; creencias culturales y religiosas; formas comunales en lugar de la propiedad individual de los medios de producción; sistemas sociales basados en relaciones de reciprocidad; y prácticas de distribución e intercambio de apoyo. Es decir, el bienestar se concibe como el derecho a vivir de forma diferente a una aparente sociedad mayoritaria que enfatiza la dimensión material del bienestar. Para ello se asume un reconocimiento a una ciudadanía diferenciada frente al resto de la sociedad.

Por otro lado, el bienestar no se limita al derecho a mantener prácticas autóctonas de redes de reciprocidad y capacidad de autogestión; también implica el derecho a construir relaciones de cooperación con el Estado y los no-Rarámuri. Esto requiere de oportunidades de generación de ingresos; acceso a los servicios básicos, y los beneficios de los programas de protección social. En este sentido, los portadores de lo que he llamado aquí el discurso subalterno están articulando la exigencia de distribuir oportunidades para participar de manera más intensa y amplia en la económica y política nacional en igualdad de circunstancias, sin las características condiciones actuales de discriminación.

Es dentro de la fluctuación entre estas dos fuerzas que la noción de bienestar toma sentido para los Rarámuri. En ciertos momentos fluctúa haciendo hincapié en elementos propios de uno u otro discurso. Pareciera que su idea de bienestar consiste precisamente en mantener este equilibrio. Gestionar el equilibrio en la fluctuación permite posicionar demandas en términos de reconocimiento étnico distintivo y en términos de redistribución de derechos y oportunidades.

\section{Reflexiones Metodológicas}

De igual manera, la evidencia aquí presentada permite reflexionar sobre las implicaciones metodológicas para el trabajo de campo etnográfico. Es decir, ¿cómo lograr que el etnógrafo sea capaz de documentar la pluralidad de voces y, crucialmente, escuchar la voz del subalterno? En el caso aquí presentado, no es el subalterno indígena necesariamente, sino de los grupos subalternizados dentro de las propias comunidades indígenas. Varias consideraciones pueden ser mencionadas al respecto. En primer lugar, la pregunta sobre la representación ha sido abordada desde los estudios subalternos por Spivak (1988) quien deconstruye lo "subalterno" como categoría monolítica en la que se presume una 
identidad y conciencia unitaria del sujeto. Haciendo eco de esta idea, creo que no debemos de caer en el supuesto de que los portadores del discurso subalterno aquí presentado tengan una identidad, conciencia o intereses homogéneos. En ese sentido es importante recordar que entiendo por lo subalterno y dominante como categorías únicamente referentes a las posiciones que guardan entre sí los debates identificados, y no así los sujetos o sus relaciones.

En segundo lugar, uno podría preguntarse sobre los problemas que implica al etnógrafo documentar el hidden transcript, sobre la posibilidad o no de "escuchar la voz del subalterno" en caso de que el subalterno en realidad "pueda hablar" (Spivak 1988). Si bien Spivak, habla en el contexto de los estudios poscoloniales -y los intelectuales y académicos en general-, que terminan reproduciendo esquemas de dominación neo-coloniales, su crítica es muy útil en este artículo para evitar caer en las redes del discurso dominante al interior de la comunidad indígena. En este sentido, Spivak resalta los peligros del trabajo intelectual que actúa, consciente o inconscientemente, a favor de la dominación del subalterno, manteniéndolo en silencio sin darle un espacio o una posición desde la que pueda "hablar". Por ello, en su opinión, el intelectual no debe -ni puede- hablar "por" el subalterno sin que esto implique proteger y reforzar las relaciones de opresión.

De la misma manera, considero que los estudios de bienestar, sobre todo aquellos de corte etnográfico, deben de basarse en la necesidad de reflexionar sobre el método utilizado para documentar y hacer visibles relaciones de poder en el contexto donde estemos insertos. Incluso, argumento que la misma definición que propongamos de bienestar, depende de las propias formas de aproximación y métodos elegidos. Asumiendo que las interacciones humanas son siempre dinámicas, construyéndose constantemente, impregnadas de relaciones de poder y posiciones desiguales, es vital considerar nuestra posicionalidad como investigadores siendo sensibles a nuestra incursión en un entorno social y cultural preexistente. En dicha incursión al campo muy posiblemente se nos asocie con figuras de poder locales: autoridades tradicionales, organizaciones no-gubernamentales, o instituciones oficiales que puedan influir en cómo somos percibidos por nuestros interlocutores y la información a la cual tengamos acceso.

Finalmente, y considerando los retos metodológicos enunciados antes, esta experiencia apela a la utilidad de los métodos etnográficos en la investigación cualitativa para explorar la política de las representaciones del bienestar. Los métodos etnográficos proporcionan profundidad a la exploración de una amplia gama de órdenes sociales. Al reconocer la pluralidad de voces sobre bienestar, podemos evitar que los órdenes sociales dominantes legitimen las asimetrías de poder. La heterogeneidad de las experiencias humanas y los métodos de investigación que usamos para documentarlas, construyen discursos de bienestar.

Agradecimientos: Se agradece el apoyo del CONACYT México por el financiamiento a la investigación que da pie a la elaboración del presente artículo, igualmente se agradece a los evaluadores y a todos quiénes lo hicieron posible.

\section{Referencias Citadas}

Chindarkar, N. 2014. ¿Is subjective well-being of concern to potential migrants from Latin America? Social Indicators Research 115:159-182

Cooke, B. y U. Kothari (eds.) 2001. Participation: The New Tyranny? Zed Books, London- New York.

Fals-Borda, O. y M.A. Rahman 1991. Action and Knowledge: Breaking the Monopoly with Participation Action-Research. Intermediate Technology Publications and Apex Press, New York.

Fraser, N. 1992. Rethinking the Public Sphere: A Contribution to the Critique of Actually Existing Democracy. En Habermas and the Public Sphere, editado por C. Calhoun, 109-142. MIT Press, Cambridge.

Fraser, N. 1997. Justice Interruptus: Critical Reflections on the Postsocialist Condition. Routledge, London.

Gudeman, S. y A. Rivera 1990. Conversations in Colombia. The Domestic Economy in Life and Text. Cambridge University Press, Cambridge.
Gudynas, E. 2011. Buen Vivir: germinando alternativas al desarrollo. América Latina en Movimiento No 462. Agencia Latino Americana Información ALAI, Quito.

Huanacuni Mamani, F. 2010. Buen Vivir Nivir Bien. Filosófica, Políticas, Estrategias y Experiencias Regionales Andinas. Coordinadora Andina de Organizaciones Indígenas (CAOI), La Paz.

Instituto Nacional de Estadística y Geografía 2015. Tabulados de la Encuesta Intercensal 2015, México, D.F.

Kennedy, J. 1963. Tesguino Complex: The role of beer in the Tarahumara culture. American Anthropologist 65:620-640.

Kennedy, J. 1978. Tarahumara of the Sierra Madre: Beer, Ecology and Social Organisation, AMH Publishing Corporation, Arlington Heights.

Loera-Gonzalez, J. 2013. Conflicting Paths to Wellbeing: Rarámuri and Mestizo inter-ethnic relations in Northern Mexico. $\mathrm{PhD}$ Thesis in Development Studies, Institute of Development Studies, University of Sussex, Brighton. 
Loera-Gonzalez, J. 2014. The Power of wellbeing discourses among indigenous and non-Indigenous people in Mexico. Institute of Development Studies Bulletin 45:29-42.

Loera-Gonzalez, J. 2015. La construcción de los buenos vivires; entre los márgenes y tensiones ontológicas. Polis, Revista Latinoamericana 40.

Mathews, G. y C. Izquierdo 2009. Pursuits of Happiness. Well-Being in Anthropological Perspective. Berghahn Books, New York-Oxford.

Saucedo, E. 2003. Reciprocidad y vida social en la Tarahumara: El Complejo del Tesguino y los grupos del sur de la Sierra. En Etnología de las regiones Indígenas de México en el Nuevo Milenio: La comunidad sin Límites, editado por S. Millán y J. Valle, Vol. III pp. 217-267, INAH, México, D.F.

Scott, J.C 1990. Domination and the Arts of Resistance: Hidden Transcripts. Yale University Press, New Haven.

Spivak, G. 1987. In Other Worlds. Essays in Cultural Politics. Methuen, New York.

Spivak, G. 1988. Can the Subaltern Speak? En Marxism and the Interpretation of Culture, editado por C. Nelson y L. Grossberg, pp. 271-313. University of Illinois Press, Urbana and Chicago.
Toledo, V. 2015. Ecocidio en México. Grijalbo, México, D.F.

United Nations Development Program 2010. Informe sobre Desarrollo Humano de los Pueblos Indígenas de México. El reto de la desigualdad de oportunidades, México, DF.

Urteaga, A. 1998. We semati ricuri: trabajo y tesgüino en la Sierra Tarahumara. En Historia General de Chihuahua v Periodo Contemporáneo: Trabajo, Territorio y Sociedad en Chihuahua durante el Siglo XX, editado por J.L. Sariego, pp. 515-527, Universidad Autónoma de Ciudad Juárez, México, DF.

Villanueva, V.H. 2006. Sistemas Políticos Indígenas: Autonomía y Libre Determinación. Aboreachi: Un Caso en la Sierra Tarahumara. Tesis (Licenciatura en Antropología), Escuela Nacional de Antropología e Historia, Chihuahua.

Virtanen, P.K. 2012. Indigenous Youth in Brazilian Amazonia: Changing Lived Worlds. Palgrave Macmillan, New York.

White S.C. y J. Pettit 2004. Participatory Approaches and the Measurement of Human Well-being. WeD Working Paper 08. ESRC Research Group on Wellbeing in Development Countries.

Wright, K. 2012. International Migration, Development and Human Wellbeing. Palgrave Macmillan.

\section{Notas}

${ }^{1}$ Defino como discursos de bienestar a aquellas representaciones, ideas y hábitos que ubican, cultural e históricamente, significados de bienestar promulgados por medio de experiencias individuales o colectivas.

${ }^{2}$ Población de tres años y más hablante de una lengua indígena.

${ }^{3}$ El término mestizo se utiliza aquí como categoría descriptiva usado por los mismos habitantes locales y que hace referencia a la población habitante de la misma región y que no es indígena.

${ }^{4}$ El teswuino también está intrínsecamente relacionado con el ciclo agrícola y forma parte de las redes de apoyo y reciprocidad social Rarámuri.
${ }^{5}$ Reconozco que, no obstante, mi edad y género significaba que seguía habiendo algunos discursos que estaban más visibles para mí que otros. Por ejemplo, soy consciente de que era mucho más fácil para mí aproximarme y generar mayor confianza con los varones que con las mujeres.

${ }^{6}$ Para un análisis detallado del sistema político Rarámuri en la región de Aboreachi ver Villanueva (2006).

${ }^{7}$ Ver Virtanen (2012) sobre cómo los jóvenes indígenas amazónicos crean sus mundos sociales a diferencia de otros miembros de las localidades, y la necesidad de incorporar sus voces silenciosas en la literatura antropológica existente. 